\title{
Switching to aflibercept among patients with treatment-resistant neovascular age-related macular degeneration: a systematic review with meta-analysis
}

\author{
This article was published in the following Dove Press journal: \\ Clinical Ophthalmology \\ 6 January 2017 \\ Number of times this article has been viewed
}

\author{
Kimberly Spooner ${ }^{1-3}$ \\ Thomas Hong ${ }^{1,2}$ \\ Wijeyanthy Wijeyakumar ${ }^{1-3}$ \\ Andrew A Chang ${ }^{1-3}$ \\ 'Sydney Retina Clinic \& Day \\ Surgery, ${ }^{2}$ Sydney Institute of Vision \\ Science, Sydney, ${ }^{3}$ Save Sight Institute, \\ Department of Ophthalmology, \\ University of Sydney, Camperdown, \\ NSW, Australia
}

\begin{abstract}
Purpose: To systematically review anatomical and functional outcomes subsequent to switching from bevacizumab/ranibizumab to aflibercept monotherapy in patients with treatment-resistant neovascular age-related macular degeneration (nAMD).

Design: Systematic review and meta-analysis.

Methods: Medline, PubMed, Embase, and Cochrane databases were searched up to July 2016 for available scientific literature which met inclusion criteria. Eligible studies reported visual and anatomical outcomes with at least 6 months of follow-up among patients with nAMD and persistent or resistant exudative fluid despite previous anti-vascular endothelial growth factor (VEGF) therapy (bevacizumab and/or ranibizumab) and were switched to aflibercept monotherapy. Mean changes in best-corrected visual acuity (BCVA) and central retinal thickness (CRT) were pooled using random-effects models with 95\% confidence intervals (CIs).

Results: Of 82 papers reviewed, 28 studies met inclusion criteria of this review. Pooled results showed a small mean improvement in BCVA at 6 and 12 months following switching (1.11 letters, $95 \% \mathrm{CI}-0.25$ to $2.46, P=0.17$ and 0.63 letters, $95 \% \mathrm{CI}-0.26$ to $1.52, P=0.17$, respectively). There was a significant improvement in mean CRT following switching $(-61.90 \mu \mathrm{m}$, $95 \% \mathrm{CI}-77.10$ to $-46.80, P<0.001$ and $-50.00 \mu \mathrm{m}, 95 \% \mathrm{CI}-63.20$ to $-36.80, P<0.001$ at 6 and 12 months, respectively).
\end{abstract}

Conclusion: Pooled analysis demonstrated significantly improved anatomical outcomes; however, visual function remained stable, having a comparable effect to other anti-VEGF agents in preservation of vision. These patients had poorly responsive chronic disease with limited potential for visual recovery. Switching to aflibercept with frequent monitoring may be a suitable option for patients who have developed treatment resistance.

Keywords: anti-vascular endothelial growth factor, macular degeneration, treatment resistance, meta-analysis, aflibercept

\section{Introduction}

Age-related macular degeneration (AMD) is a progressive, degenerative disease of the retina which is the leading cause of blindness among older people in western countries. ${ }^{1-8}$ There are 2 main forms of late-stage AMD; "dry" AMD is characterized by the development of either drusen, pigmentary changes or atrophy of the retinal pigment epithelium (RPE) in the macular region (geographic atrophy, GA). Aside from antioxidant therapy, there are no current treatments for dry AMD, although numerous clinical trials are under development. ${ }^{9}$ Wet AMD or neovascular AMD (nAMD) is
Correspondence: Andrew A Chang Sydney Retina Clinic \& Day Surgery, Level I3, Park House, I87 Macquarie Street, Sydney, NSW 2000, Australia Tel +6I 2 922I 3755

Fax +6I 292211637

Email achang@sydneyretina.com.au 
characterized by choroidal neovascularization (CNV), an anomalous angiogenic process controlled by growth factors, including vascular endothelial growth factor (VEGF), placental growth factor (PlGF), platelet-derived growth factor, insulin-like growth factor, and angiogenic inhibitors such as pigment epithelium-derived factor. ${ }^{10,11}$ These choroidal neovascular vessels typically leak and bleed, leading to intra- or subretinal fluid, and lipid exudation which may result in severe vision loss. ${ }^{7}$ Without treatment, the process usually evolves into fibrous scarring greatly diminishing visual capacity. ${ }^{12}$

Until the introduction of anti-VEGF agents, treatments most commonly used for nAMD included thermal laser photocoagulation and verteporfin photodynamic therapy (PDT). ${ }^{8}$ However, neither laser photocoagulation nor PDT offered any significant potential for visual recovery when the central macula is involved. Anti-VEGF therapy has become the standard treatment for $\mathrm{nAMD}$ with several anti-VEGF agents available: Avastin ${ }^{\circledR}$ (bevacizumab), Lucentis ${ }^{\circledR}$ (ranibizumab), and more recently Eylea ${ }^{\circledR}$ (aflibercept). Both ranibizumab and aflibercept have been approved by the US Food and Drug Administration (FDA) for use in the eye. ${ }^{13,14}$ Anti-VEGF therapy has been shown to be effective in the treatment of a number of other ocular diseases, such as diabetic macular edema, and retinal vein occlusions.

Aflibercept, previously known as VEGF Trap (Regeneron Pharmaceuticals, Inc., Tarrytown, NY, USA), is the most recent anti-VEGF agent; the molecule serves as a soluble VEGF decoy to impede the growth of new blood vessels by binding to VEGF-A (with a higher affinity than its natural receptors, and both ranibizumab and bevacizumab), VEGF-B, and PlGF. It is therefore thought to be more effective in blocking VEGF and has been effective in naïve eyes $^{15}$ and was approved for the treatment of nAMD by the FDA in 2011. ${ }^{14}$

Long-term, repeated use of ranibizumab and bevacizumab has been associated with diminished effectiveness. A significant proportion of patients treated with these therapies, termed "nonresponders", do not benefit from continued treatment, and vision continues to deteriorate. ${ }^{16-18}$ Therapeutic options for nonresponders are limited to offering a different therapeutic agent, combining therapy, or decreasing treatment intervals. With up to $25 \%$ of patients classed as nonresponders, ${ }^{16}$ there is a greater need for understanding of treatment resistance to improve treatment strategies.

The purpose of this review is to investigate the functional and anatomical response as well as the effect of differing treatment regimens, and disease chronicity among nAMD patients (resistant or refractory) switched to aflibercept previously treated with other intravitreal anti-VEGF agents (ranibizumab and bevacizumab).

\section{Methods}

\section{Search strategy}

A search of Medline, PubMed, Embase, and Cochrane databases was undertaken independently by 2 reviewers (KS and $\mathrm{TH})$. The search strategy was based on the combination of medical subject headings and the keywords "neovascular AMD", "wet AMD treatment", "aflibercept", "Eylea", "age-related macular degeneration", “ARMD”, "switching", "refractory", "anti-vascular endothelial growth factor", and "anti-VEGF". Studies published from January 2012 to July 2016 were included in this review. The citations of related articles were examined for additional publications.

When data was missing, unclear, or incomplete, we attempted to contact study authors for clarification and additional information.

\section{Eligibility criteria}

This review considered studies that included populations that had undergone previous anti-VEGF therapy with either ranibizumab and/or bevacizumab, before being switched to aflibercept monotherapy due to persistent or resistant exudative fluid secondary to nAMD.

We limited the search to clinical studies published in peer-reviewed, and English language publications. Studies were excluded if participants were not followed for a minimum of 6 months or when only an abstract was published. The key inclusion criteria were the following: (a) patients should be aged 50 years or older with active subfoveal CNV lesions secondary to AMD, who are resistant to previous anti-VEGF therapy; and (b) Early Treatment Diabetic Retinopathy Study (ETDRS) best-corrected visual acuity (BCVA) should be between 75 and 25 letters (20/32 to 20/320 Snellen equivalent).

\section{Study selection}

The review authors performed the literature search by screening citations by title and abstract, identified all studies for full review to confirm eligibility, and selected independent studies for their inclusion in the systematic review. Any disagreement between these reviewers was resolved by a third reviewer (AC), and the decision to include and exclude a study was reached by consensus.

\section{Data extraction and quality assessment}

The review authors independently screened articles, extracted data, and assessed risks of bias, following the 27-point 
Preferred Reporting Items for Systematic Reviews and Meta-Analyses (PRISMA) checklist. The review authors then independently assessed the full-text reports and removed articles that were not relevant to the topic.

The authors extracted data and tabulated data such as mean change of visual acuity (VA), central retinal thickness (CRT), and pigment epithelial detachment (PED) height from baseline. When extracting data, the Cochrane handbook was used to obtain standard deviation from range, median, or $P$-value when present. ${ }^{19}$ BCVA data was converted to ETDRS letter score.

Data were summarized both qualitatively and quantitatively. This was facilitated by extracting data in regard to the following characteristics from all included studies: study design (retrospective or prospective), study setting (country), patient selection (target population, sample size), patient characteristics (age, gender), and clinical characteristics (BCVA, CRT, PED height, dosing regimens).

Further to the overall meta-analysis, we also stratified the studies by the number of previous anti-VEGF therapies before conversion to aflibercept ( $<12$ injections, 12-23 injections, and $\geq 24$ injections), the number of aflibercept injections received during the course of the study $(<4$ injections and $\geq 4$ injections for the 6-month studies and $<8$ injections and $\geq 8$ injections for the 12-month studies), study design type (robustness of prospective vs retrospective studies), and dosing regimen during the course of the study. If studies had missing data regarding the stratification, this was removed from the analysis for the corresponding meta-analysis.

The review authors independently assessed potential sources of bias according to the Downs \& Black Checklist. ${ }^{20}$ All included studies were assessed as fair. Insufficient information was presented in many cases to enable us to assess the quality and strength of studies in 2 domains: measures taken to reduce bias $(n=25)$ and patients lost to follow-up $(n=24)$.

\section{Data synthesis and analysis}

A meta-analysis of eligible studies was conducted using Comprehensive Meta-Analysis Version 2.0 (Biostat, Englewood, NJ, USA). All results were subjected to double data entry, and effect sizes were expressed as mean differences (for continuous data) with 95\% confidence intervals (CIs). Results were pooled using random-effects models and visualized in forest plots. Meta-regression analysis was used to identify heterogeneity and quality factors that were subsequently used to stratify pooled estimates. Heterogeneity was assessed with the $I^{2}$ statistic, which is the percentage of between-study heterogeneity that is attributable to variability in the true effect, with $25 \%, 50 \%$, and $>75 \%$ indicating low, moderate, and high heterogeneity, respectively. ${ }^{21}$ In cases where heterogeneity was significant, a random-effects model was used. Publication bias was assessed with funnel plots and Egger's linear regression.

\section{Results \\ Description of studies}

Of the 82 articles identified by the initial search, 43 were found to be potentially relevant for the review. Of these, 15 were excluded - 1 study shared the same sample and time period, 3 assessed polypoidal choroidal vasculopathy rather than $\mathrm{CNV}$ lesions, 6 were under the desired time of follow-up, and 5 had insufficient data.

Twenty-eight studies (Table 1) that met the inclusion criteria for the review were identified. All studies examined the efficacy and safety of aflibercept for patients with nAMD previously treated with bevacizumab and/or ranibizumab. Sample sizes ranged from 11 to 447, with a total of 2,254 eyes included in this meta-analysis. The study duration ranged from 6 to 24 months. The literature search process and reasons for exclusion are summarized in Figure 1.

The majority of included studies reported at least 1 functional and anatomical measure. Reported outcomes included BCVA changes and stability, changes in CRT, macular volume, and PED height. Overall, we included 8 prospective p2-29 $^{22}$ $20^{30-49}$ retrospective studies, with a mean follow-up of at least 6 months after converting to aflibercept. Two articles included the same eyes at different times after conversion. ${ }^{23,24}$

While we were able to identify more studies that examined the effect of switching to aflibercept, insufficient outcome data prevented them from being included in this meta-analysis.

\section{Baseline characteristics}

Baseline characteristics of the patients included within the individual studies were similar across all studies (Tables 1 and 2). The mean age ranged from 70.1 to 83.4 years, and $59 \%$ of patients were female. The mean baseline BCVA letter scores ranged from 42.50 to 74.20 ETDRS letters and were similar among the studies. The mean CRT ranged from 228.60 to $449.00 \mu \mathrm{m}$. The mean PED height ranged from 122.80 to $313.00 \mu \mathrm{m}$.

\section{Meta-analysis}

Best-corrected visual acuity

Nineteen studies ( $n=1,434$ eyes) were included in the assessment of BCVA change between baseline and 6 months. Overall, the pooled results did not find a significant increase 
Table I Characteristics of included studies in the meta-analysis

\begin{tabular}{|c|c|c|c|c|c|c|}
\hline Study & Country & $\begin{array}{l}\text { Study } \\
\text { design }\end{array}$ & $\begin{array}{l}\text { Sample } \\
\text { size }\end{array}$ & $\begin{array}{l}\text { Definition of } \\
\text { treatment resistance }\end{array}$ & Inclusion/exclusion criteria & $\begin{array}{l}\text { Mean follow-up } \\
\text { (months) }\end{array}$ \\
\hline Aghdam et $\mathrm{al}^{25}$ & Germany & Prospective & 22 & $\begin{array}{l}\text { Persistent fluid after } \\
>6 \text { months of monthly } \\
\text { ranibizumab therapy }\end{array}$ & $\begin{array}{l}\text { No previous macular laser, PDT, } \\
\text { or vitrectomy } \\
\text { No history of RAP/PCV or } \\
\text { presence of macular fibrosis scar }\end{array}$ & 12 \\
\hline Arcinue et $\mathrm{a}^{30}$ & USA & Retrospective & 63 & $\begin{array}{l}\text { Persistent retinal fluid } \\
\text { after the eyes have been } \\
\text { dry following at least } 3 \\
\text { injections every } 4 \text { weeks; } \\
\text { or persistence of } \\
\text { exudation or fibrovascular } \\
\text { PED with IRF/SRF } \\
\text { on OCT while on } \\
\text { monthly ranibizumab or } \\
\text { bevacizumab monotherapy }\end{array}$ & $\begin{array}{l}\text { Excluded if they had received } \\
\text { aflibercept elsewhere prior; } \\
\text { macular hole, VMT, ERM, retinal } \\
\text { detachment, PCV, macular } \\
\text { dystrophy, or peripapillary CNV }\end{array}$ & 12 \\
\hline Bakall et $\mathrm{a}^{42}$ & USA & Retrospective & 36 & $\begin{array}{l}\text { Persistent IRF/SRF despite } \\
\text { monthly intravitreal } \\
\text { injections with the same } \\
\text { drug or a change from } \\
\text { either bevacizumab or } \\
\text { ranibizumab to the other } \\
4 \text { weeks apart }\end{array}$ & $\begin{array}{l}\text { IRF/SRF present for minimum of } \\
3 \text { months prior to baseline } \\
\text { Must be treated with minimum } \\
3 \text { aflibercept injections }\end{array}$ & 6 \\
\hline $\begin{array}{l}\text { Barthelmes } \\
\text { et } \mathrm{al}^{31}\end{array}$ & Australia & Retrospective & 384 & $\begin{array}{l}\text { Persistent exudation and } \\
\text { loss of vision despite } \\
\text { regular treatment }\end{array}$ & $\begin{array}{l}\text { At least } 12 \text { months previously } \\
\text { treated with ranibizumab and } \\
12 \text { months of follow-up after } \\
\text { switch }\end{array}$ & 12 \\
\hline $\begin{array}{l}\text { Broadhead } \\
\text { et } \mathrm{al}^{22}\end{array}$ & Australia & Prospective & 43 & $\begin{array}{l}\text { Persistent IRF or SRF and } \\
\text { PED on OCT for at least } \\
6 \text { months despite at least } \\
4 \text { intravitreal anti-VEGF } \\
\text { injections in the past }\end{array}$ & $\begin{array}{l}\text { BCVA of } 35-90 \text { letters } \\
(20 / 200-20 / 20) \\
\text { PED height }>100 \mu \mathrm{m}\end{array}$ & 12 \\
\hline Chan et $\mathrm{a}^{32}$ & USA & Retrospective & 189 & $\begin{array}{l}\text { Persistent or recurrent } \\
\text { macular edema, SRF, } \\
\text { hemorrhage, exudates, } \\
\text { and/or PED to the same } \\
\text { drugs during the time } \\
\text { of drug transition to } \\
\text { aflibercept }\end{array}$ & $\begin{array}{l}\text { Received at least } 3 \text { consecutive } \\
\text { injections of the same anti-VEGF } \\
\text { prior (bevacizumab and/or } \\
\text { ranibizumab) to baseline } \\
\text { Must receive } 3 \text { loading doses and } \\
\text { have } 6 \text { months of follow-up }\end{array}$ & 6 \\
\hline Chang et $\mathrm{a}^{24}$ & Australia & Prospective & 49 & $\begin{array}{l}\text { Persistent IRF/SRF on } \\
\text { OCT despite at least } 4 \\
\text { anti-VEGF injections in the } \\
\text { previous } 6 \text { months }\end{array}$ & $\begin{array}{l}\text { BCVA of } 35-90 \text { letters } \\
(20 / 200-20 / 20) \\
\text { Excluded if: uncontrolled } \\
\text { IOP }>25 \mathrm{mmHg} \text {, h/o vitreous } \\
\text { hemorrhage, inflammation, } \\
\text { vitrectomy, PDT, or subfoveal } \\
\text { atrophy; triamcinolone } 180 \text { days } \\
\text { prior to baseline }\end{array}$ & 6 \\
\hline Chang et $\mathrm{a}^{23}$ & Australia & Prospective & 49 & $\begin{array}{l}\text { Presence of IRF/SRF } \\
\text { OCT despite at least } 4 \\
\text { intravitreal injections of } \\
\text { an anti-VEGF agent in the } \\
\text { preceding } 6 \text { months }\end{array}$ & $\begin{array}{l}\text { BCVA of } 35-90 \text { letters } \\
\text { (20/200-20/20) } \\
\text { Excluded if: uncontrolled } \\
\text { IOP }>25 \mathrm{mmHg} \text {; h/o vitreous } \\
\text { hemorrhage, inflammation, } \\
\text { vitrectomy, PDT, or subfoveal } \\
\text { atrophy; triamcinolone } 180 \text { days } \\
\text { prior to baseline }\end{array}$ & 12 \\
\hline
\end{tabular}


Table I (Continued)

\begin{tabular}{|c|c|c|c|c|c|c|}
\hline Study & Country & $\begin{array}{l}\text { Study } \\
\text { design }\end{array}$ & $\begin{array}{l}\text { Sample } \\
\text { size }\end{array}$ & $\begin{array}{l}\text { Definition of } \\
\text { treatment resistance }\end{array}$ & Inclusion/exclusion criteria & $\begin{array}{l}\text { Mean follow-up } \\
\text { (months) }\end{array}$ \\
\hline $\begin{array}{l}\text { Chatziralli } \\
\text { et } \mathrm{al}^{43}\end{array}$ & UK & Retrospective & 447 & $\begin{array}{l}\text { Persisting or increasing } \\
\text { SRF/IRF or PED on } \\
\text { OCT or the presence of } \\
\text { macular hemorrhage }\end{array}$ & $\begin{array}{l}\text { Received at least } 3 \text { monthly } \\
\text { ranibizumab injections over a } \\
\text { period of no more than } 4 \text { months } \\
\text { before switch } \\
\text { No h/o vitrectomy, prior PDT, } \\
\text { or laser treatment; uncontrolled } \\
\text { glaucoma; or uveitis }\end{array}$ & 12 \\
\hline Cho et $\mathrm{al}^{41}$ & USA & Retrospective & 28 & $\begin{array}{l}\text { Persistent IRF/SRF } \\
28-35 \text { days after a } \\
\text { minimum of } 6 \text { ranibizumab } \\
\text { and/or bevacizumab } \\
\text { injections }\end{array}$ & $\begin{array}{l}\text { Excluded if: OCT was dry at any } \\
\text { time } 3 \text { months prior to baseline; } \\
\text { OCT or FFA suggesting retinal } \\
\text { tubulation without IRF/SRF or } \\
\text { cystic degeneration }\end{array}$ & 6 \\
\hline $\begin{array}{l}\text { de } \\
\text { Massougnes } \\
\text { et } \mathrm{al}^{33}\end{array}$ & Switzerland & Retrospective & 60 & $\begin{array}{l}\text { Presence of IRF/SRF at } \\
\text { each visit I month after } \\
\text { injection to ranibizumab } \\
\text { treatment during } \\
9 \text { months leading to switch }\end{array}$ & $\begin{array}{l}\text { PED height }>150 \mu \mathrm{m} \\
\text { Excluded if: PCV, or any other } \\
\text { confounding retinal pathology }\end{array}$ & 9 \\
\hline Eadie et $\mathrm{al}^{34}$ & USA & Retrospective & 67 & $\begin{array}{l}\text { Persistent exudation } \\
\text { confirmed on OCT } \\
\text { despite a minimum of } 3 \\
\text { anti-VEGF injections }\end{array}$ & $\begin{array}{l}\text { Nil retinal thickening due to } \\
\text { subretinal fibrosis }\end{array}$ & 24 \\
\hline Gerding $^{45}$ & Switzerland & Retrospective & 40 & $\begin{array}{l}\text { The necessity of monthly } \\
\text { ranibizumab injections, } \\
\text { or OCT findings were } \\
\text { worse within the last } 6 \\
\text { months than previously } \\
\text { under an equal or lower } \\
\text { frequency of ranibizumab } \\
\text { treatment }\end{array}$ & $\begin{array}{l}\text { No other treatment for AMD. } \\
\text { Completed } 6 \text { months of follow-up }\end{array}$ & 6 \\
\hline $\begin{array}{l}\text { Gharbiya } \\
\text { et } \mathrm{al}^{35}\end{array}$ & Italy & Retrospective & 31 & $\begin{array}{l}\text { Persistent IRF/SRF despite } \\
\text { at least } 6 \text { consecutive } \\
\text { monthly injections with } \\
\text { ranibizumab }\end{array}$ & $\begin{array}{l}\text { Interval between last injection } \\
\text { and baseline not }<4 \text { weeks or } \\
\text { exceeding } 6 \text { weeks } \\
\text { Must have at least } 6 \text { months of } \\
\text { follow up } \\
\text { No prior PDT, PCV, RAP, or } \\
\text { intraocular surgery except } \\
\text { standard cataract surgery }\end{array}$ & 6 \\
\hline Grewal et al ${ }^{26}$ & USA & Prospective & 21 & $\begin{array}{l}\text { Persistent IRF/SRF or } \\
\text { PED despite }>6 \text { months } \\
\text { of monthly anti-VEGF } \\
\text { treatment }\end{array}$ & $\begin{array}{l}\text { Previously treated with at least } 6 \\
\text { anti-VEGF I } 2 \text { injections } \\
\text { No PCV, CSR, RPE tear, PDT } \\
\text { ( } 3 \text { months prior to baseline), } \\
\text { subfoveal fibrosis, or prior } \\
\text { triamcinolone (within } 6 \text { months } \\
\text { prior to baseline); intraocular } \\
\text { Sx within } 2 \text { months prior } \\
\text { to baseline; h/o vitrectomy, } \\
\text { inflammation, and subretinal } \\
\text { hemorrhage }\end{array}$ & 12 \\
\hline Hall et $\mathrm{al}^{36}$ & USA & Retrospective & 30 & $\begin{array}{l}\text { Central macular edema or } \\
\text { submacular fluid despite a } \\
\text { mean of } 19.1 \pm 0.06 \text { prior } \\
\text { to anti-VEGF injections }\end{array}$ & $\begin{array}{l}\text { Received } 2 \text { anti-VEGF injections } \\
\text { prior to baseline, and had to be } \\
\text { followed for } 6 \text { months after the } \\
\text { switch }\end{array}$ & 12 \\
\hline
\end{tabular}


Table I (Continued)

\begin{tabular}{|c|c|c|c|c|c|c|}
\hline Study & Country & $\begin{array}{l}\text { Study } \\
\text { design }\end{array}$ & $\begin{array}{l}\text { Sample } \\
\text { size }\end{array}$ & $\begin{array}{l}\text { Definition of } \\
\text { treatment resistance }\end{array}$ & Inclusion/exclusion criteria & $\begin{array}{l}\text { Mean follow-up } \\
\text { (months) }\end{array}$ \\
\hline Hariri et $\mathrm{a}^{46}$ & USA & Retrospective & 142 & $\begin{array}{l}\text { Refractory fluid to other } \\
\text { anti-VEGF therapies }\end{array}$ & $\begin{array}{l}\text { At least } 3 \text { injections in the } \\
\text { previous } 12 \text { months prior to } \\
\text { baseline } \\
\text { No DMO, CRVO, ERM, CSR, } \\
\text { previous inflammation, or PDT }\end{array}$ & 12 \\
\hline He et al ${ }^{17}$ & USA & Retrospective & 142 & $\begin{array}{l}\text { Refractory fluid to other } \\
\text { anti-VEGF therapies }\end{array}$ & $\begin{array}{l}\text { At least } 3 \text { injections in the previous } \\
12 \text { months prior to baseline } \\
\text { No DMO, CRVO, ERM, CSR, } \\
\text { previous inflammation, PDT }\end{array}$ & 12 \\
\hline $\begin{array}{l}\text { Kanesa- } \\
\text { Thasan et } \mathrm{al}^{37}\end{array}$ & USA & Retrospective & 11 & $\begin{array}{l}\text { Persistent PED, IRF/SRF, } \\
\text { and/or sub-RPE fluid } \\
\text { with adjacent SRF or IRF } \\
\text { after at least } 6 \text { months } \\
\text { of monthly anti-VEGF } \\
\text { treatment }\end{array}$ & $\begin{array}{l}\text { No PDT, subfoveal fibrosis, h/o } \\
\text { vitrectomy, active inflammation, } \\
\text { vitreous hemorrhage, subretinal } \\
\text { hemorrhage involving at least I } \\
\text { disc area of central fovea, previous } \\
\text { RPE tear, or PCV and CSR }\end{array}$ & 18 \\
\hline Kumar et $\mathrm{a}^{38}$ & USA & Retrospective & 34 & $\begin{array}{l}\text { Persistent IRF/SRF or } \\
\text { subfoveal sub-RPE fluid } \\
\text { with adjacent IRF/SRF fluid } \\
\text { on OCT despite at least } 3 \\
\text { consecutive injections } \\
\text { with ranibizumab }\end{array}$ & $\begin{array}{l}\text { Had consecutive anti-VEGF } \\
\text { therapy in } 6 \text { months prior to } \\
\text { baseline ( } 3 \text { being ranibizumab) } \\
\text { Interval between the last } \\
\text { ranibizumab injection and baseline } \\
\text { not exceeding } 42 \text { days }\end{array}$ & 6 \\
\hline Major et $\mathrm{al}^{48}$ & USA & Retrospective & 60 & $\begin{array}{l}\text { Persistent PED despite } \geq 2 \\
\text { prior anti-VEGF injections }\end{array}$ & $\begin{array}{l}\text { No h/o CSR, glaucoma, or } \\
\text { intraocular steroids }\end{array}$ & 12 \\
\hline Mantel et $\mathrm{al}^{27}$ & Switzerland & Prospective & 21 & $\begin{array}{l}\text { Presence of IRF/SRF } \\
\text { despite monthly } \\
\text { retreatment with } \\
\text { ranibizumab }\end{array}$ & $\begin{array}{l}\text { Previous ranibizumab Rx for } \\
2 \text { years and still had persistent fluid } \\
\text { BCVA between } 20 / 25 \text { and } 20 / 400 \\
\text { Max lesion size I2 DD }\end{array}$ & 12 \\
\hline $\begin{array}{l}\text { Narayan and } \\
\text { Muecke }^{39}\end{array}$ & Australia & Retrospective & 80 & $\begin{array}{l}\text { Persistent macular fluid } \\
\text { after }>12 \text { months of } \\
\text { ranibizumab treatment }\end{array}$ & $\begin{array}{l}\text { Eyes excluded if they received } \\
\text { prior PDT }\end{array}$ & 12 \\
\hline $\begin{array}{l}\text { Pinheiro- } \\
\text { Costa et } \mathrm{a}^{44}\end{array}$ & Portugal & Retrospective & 82 & $\begin{array}{l}\text { Persistent IRF/SRF after } \\
\geq 3 \text { consecutive monthly } \\
\text { bevacizumab injections }\end{array}$ & $\begin{array}{l}\text { Minimum } 3 \text { anti-VEGF before } \\
\text { baseline } \\
\text { Received at least } 3 \text { aflibercept } \\
\text { with follow-up }\end{array}$ & 8.3 \\
\hline Ricci et al ${ }^{49}$ & Italy & Retrospective & 72 & $\begin{array}{l}\text { Persistent IRF/SRF } \\
\text { after at least } 3 \text { monthly } \\
\text { consecutive ranibizumab } \\
\text { injections as evaluated by } \\
\text { OCT }\end{array}$ & $\begin{array}{l}\text { Exclusion criteria: Prior } \\
\text { vitrectomy, trabulectomy, surgery } \\
\text { in the study eye within } 3 \text { months } \\
\text { of baseline, uncontrolled } \\
\text { glaucoma, uveitis, or other ocular } \\
\text { disease to confound the results }\end{array}$ & 12 \\
\hline Sarao et $\mathrm{a}^{28}$ & Italy & Prospective & 92 & $\begin{array}{l}\text { Persistent or recurrent IRF/ } \\
\text { SRF on OCT after at least } \\
4 \text { intravitreal ranibizumab } \\
\text { injections during the } \\
\text { previous } 6 \text { months }\end{array}$ & $\begin{array}{l}\text { BCVA }<70 \text { ETDRS } \\
\text { No RAP, PCV, RPE tear, active } \\
\text { inflammation, or infection }\end{array}$ & 12 \\
\hline Thorell et $\mathrm{a}^{40}$ & USA & Retrospective & 73 & $\begin{array}{l}\text { Persistent or recurrent } \\
\text { IRF/SRF on OCT despite } \\
\text { at least } 12 \text { months of } \\
\text { intravitreal bevacizumab } \\
\text { or ranibizumab }\end{array}$ & $\begin{array}{l}\text { Received at least } 12 \text { months } \\
\text { of anti-VEGF therapy prior to } \\
\text { baseline } \\
\text { No concomitant retinal pathology }\end{array}$ & 6 \\
\hline Wykoff et al ${ }^{29}$ & USA & Prospective & 46 & $\begin{array}{l}\text { Recalcitrant fluid despite } \\
\text { monthly or near-monthly } \\
\text { anti-VEGF therapy }\end{array}$ & $\begin{array}{l}\text { Previously treated on 2-year } \\
\text { SAVE trial } \\
\text { No subretinal fibrosis or GA }\end{array}$ & 6 \\
\hline
\end{tabular}

Abbreviations: AMD, age-related macular degeneration; BCVA, best-corrected visual acuity; CNV, choroidal neovascularization; CRVO, central retinal vein occlusion; CSR, central serous retinopathy; DD, disc diameters; DMO, diabetic macular edema; ERM, epiretinal membrane; ETDRS, Early Treatment Diabetic Retinopathy Study; GA, geographic atrophy; FFA, fundus fluorescein angiogram; h/o, history of; IOP, intraocular pressure; IRF, intraretinal fluid; OCT, optical coherence tomography; PCV, polypoidal choroidal vasculopathy; PDT, photodynamic therapy; PED, pigment epithelial detachment; RAP, retinal angiomatous proliferation; RPE, retinal pigment epithelium; Rx, treatment; SRF, subretinal fluid; Sx, surgery; VEGF, vascular endothelial growth factor; VMT, vitreomacular traction. 


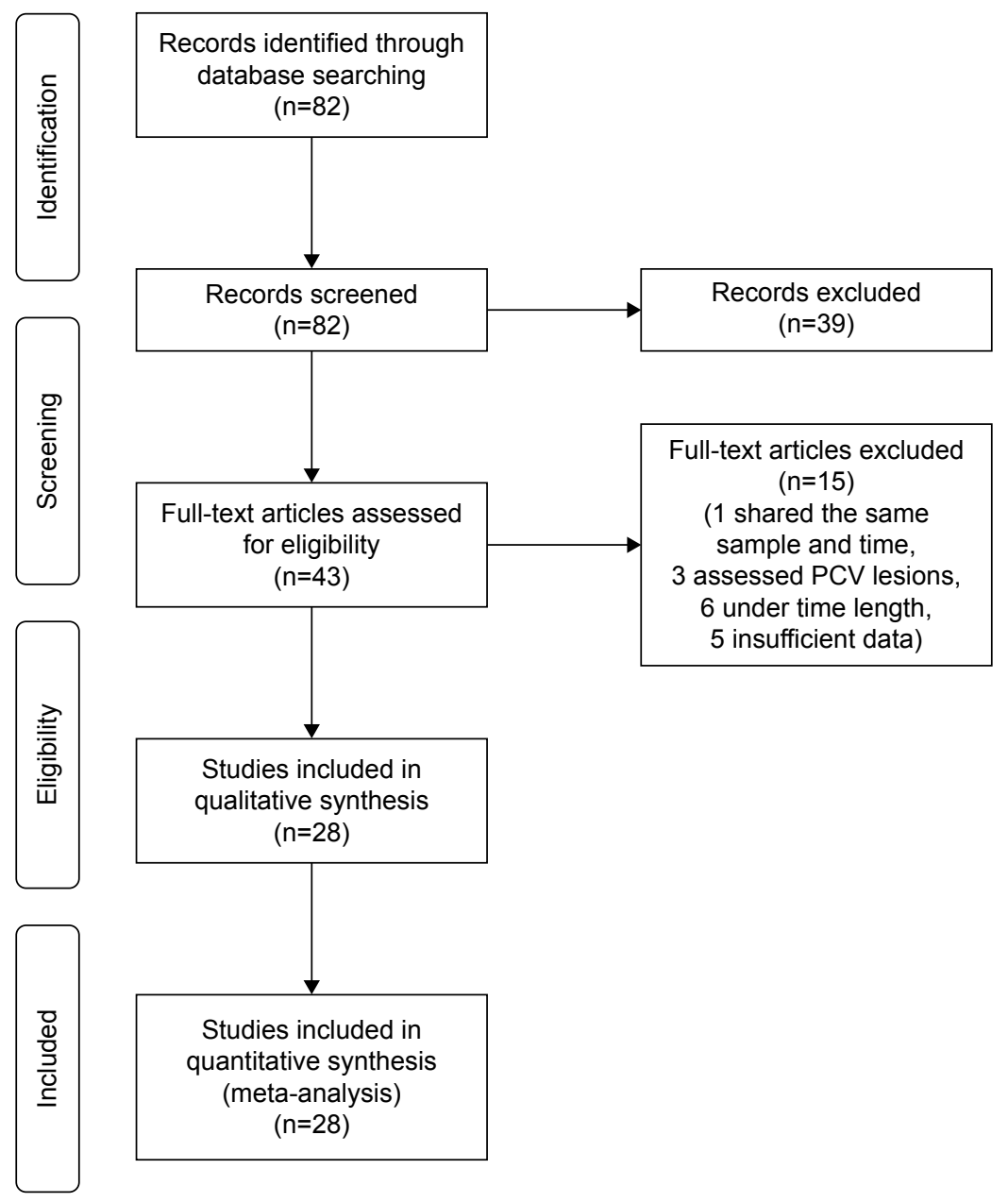

Figure I Data sample extraction.

Abbreviation: PCV, polypoidal choroidal vasculopathy.

in BCVA from baseline with a mean increase of 1.11 letters ( $95 \% \mathrm{CI}-0.25$ to $2.46, P=0.11$ ) (Figure 2).

Fifteen studies ( $\mathrm{n}=1,561$ eyes) were included in the assessment of BCVA change between baseline and 12 months. There was no significant change in BCVA from baseline with a mean increase of 0.63 letters $(95 \%$ CI -0.26 to 1.52 , $P=0.17$ ) (Figure 3). Significant heterogeneity was found across studies assessing BCVA for 6 and 12 months of follow-up $\left(I^{2}=95.85 \%, P<0.001\right.$ and $I^{2}=91.81 \%, P<0.001$, respectively).

When the studies were stratified by the number of previous injections prior to switching to aflibercept at 6 months, studies with less mean number of previous injections $(<12)$ showed a greater mean increase of 3.83 letters compared to those which had a greater mean number of previous injections $(>24)$ which showed a lesser mean increase of only 0.05 letter (Table 3). Stratification by the number of aflibercept injections showed that studies which had less injections of aflibercept over the course of the study had a greater improvement in
BCVA compared to those which had more injections over the course of the study (2.97 vs 0.49 letters at 6 months).

Studies with a pro re nata (PRN) regimen without a loading dose had the greatest mean increase in BCVA of 3.47 letters at 6 months. Studies with a regimen of 3 loading doses followed by PRN dosing had the greatest mean increase in BCVA of 2.20 (6 months) and 13.41 letters (12 months). This is compared to studies following a treat-and-extend regimen which had the lowest change in BCVA with a mean decrease of 1.20 and 0.73 letters at 6 and 12 months, respectively. There was significant change in BCVA among prospective studies, exhibiting a greater increase in mean BCVA of 3.96 and 2.79 letters at 6 and 12 months, respectively, compared to those retrospective in nature in which mean BCVA of $<1$ letter was noted at 6 and 12 months.

\section{Central retinal thickness}

Seventeen studies (1,343 eyes) were included in the metaanalysis of the mean change of CRT between baseline and 
Table 2 Clinical characteristics of included studies in the meta-analysis

\begin{tabular}{|c|c|c|c|c|c|}
\hline Study & $\begin{array}{l}\text { Mean age in } \\
\text { years (range) }\end{array}$ & $\begin{array}{l}\text { Number of injections } \\
\text { prior to conversion } \\
\text { (range) }\end{array}$ & $\begin{array}{l}\text { Time between last } \\
\text { anti-VEGF treatment } \\
\text { and conversion (range) }\end{array}$ & $\begin{array}{l}\text { Mean number of } \\
\text { aflibercept injections } \\
\text { (range) }\end{array}$ & Treatment regimen \\
\hline Aghdam et $\mathrm{a}^{25}$ & $\begin{array}{l}73.95 \pm 10.65 \\
(56-91)\end{array}$ & $9.55 \pm 6.91(6-30)$ & $\begin{array}{l}\text { Not }<4 \text { weeks or } \\
>6 \text { weeks }\end{array}$ & $7.27 \pm 1.98(4-12)$ & $\begin{array}{l}\text { Loading phase of } \\
\text { aflibercept every } \\
4 \text { weeks for } 3 \text { months } \\
\text { followed by PRN }\end{array}$ \\
\hline \multirow[t]{2}{*}{ Arcinue et $\mathrm{al}^{30}$} & $\begin{array}{l}81.00 \\
(I Q R \text { 76-87) }\end{array}$ & I 3.00 (IQR 7-22) & $\begin{array}{l}\text { Median } 6 \text { weeks } \\
\text { (range } 4-18 \text { ) }\end{array}$ & $\begin{array}{l}\text { Those treated every } \\
8 \text { weeks }=4(4-6)\end{array}$ & $\begin{array}{l}8 \text { weekly aflibercept } \\
\text { injections without loading } \\
\text { phase }\end{array}$ \\
\hline & & & & $\begin{array}{l}2 \text { I eyes were switched } \\
\text { to every } 4 \text {-weekly } \\
\text { treatment }\end{array}$ & $\begin{array}{l}\text { In case of persistent fluid } \\
\text { regimen, changed to } \\
\text { every } 4 \text { weeks }\end{array}$ \\
\hline Bakall et $a^{42}$ & $79.00(60-88)$ & $\begin{array}{l}25.60(6-74) \\
10.6 \text { for ranibizumab group } \\
\text { I5.0 for bevacizumab group }\end{array}$ & 4 weeks & $\begin{array}{l}5.72 \text { (in } 9 \text { eyes treatment } \\
\text { was stopped prior to } \\
6 \text { months: in } 8 \text { patients } \\
\text { after the fifth injection } \\
\text { and in I patient after the } \\
\text { fourth injection) }\end{array}$ & $\begin{array}{l}\text { Loading phase of } \\
\text { aflibercept every } \\
4-6 \text { weeks for } 3 \text { months, } \\
\text { followed by PRN }\end{array}$ \\
\hline Barthelmes et $\mathrm{al}^{31}$ & $\mathrm{~N} / \mathrm{A}$ & $\mathrm{N} / \mathrm{A}$ & $\mathrm{N} / \mathrm{A}$ & 6.60 & $\mathrm{~N} / \mathrm{A}$ \\
\hline Broadhead et a ${ }^{22}$ & 78.70 & 34.80 & $\mathrm{~N} / \mathrm{A}$ & 8.00 & $\begin{array}{l}\text { Loading phase of } \\
\text { aflibercept every } \\
4 \text { weeks for } 3 \text { months, } \\
\text { followed by } 8 \text { weekly } \\
\text { injections }\end{array}$ \\
\hline \multirow[t]{4}{*}{ Chan et a ${ }^{32}$} & 83.40 & $\begin{array}{l}\text { Bevacizumab group: } 4.80 \\
\text { over } 6 \text { months prior to } \\
\text { switch }\end{array}$ & $\mathrm{N} / \mathrm{A}$ & 5.40 & $\begin{array}{l}3 \text { monthly loading doses, } \\
\text { and then treat-and- } \\
\text { observe strategy }\end{array}$ \\
\hline & & $\begin{array}{l}\text { Ranibizumab group: } 8.60 \\
\text { over } 6 \text { months prior to } \\
\text { switch }\end{array}$ & & 5.40 bevacizumab group & \\
\hline & & Mixed group: 4.90 over & & 5.50 ranibizumab group & \\
\hline & & 6 months prior to switch & & 5.50 mixed group & \\
\hline Chang et $\mathrm{a}^{24}$ & $77.80 \pm 7.50$ & $34.90 \pm 16.10$ & $>30$ days & 5.00 & $\begin{array}{l}\text { Loading phase of } \\
3 \text { monthly injections plus } \\
\text { mandatory injection at } \\
\text { weeks } 16 \text { and } 24\end{array}$ \\
\hline Chang et $\mathrm{al}^{23}$ & $77.80 \pm 7.50$ & $34.90 \pm 16.10$ & 36 days & 8.00 & $\begin{array}{l}\text { Loading phase of } \\
3 \text { monthly injections } \\
\text { plus mandatory injection } \\
\text { every } 2 \text { months at } \\
\text { months } 4,6,8,10 \text {, } \\
\text { and } 12\end{array}$ \\
\hline Chatziralli et $\mathrm{al}^{43}$ & $78.60 \pm 8.20$ & $7.00 \pm 4.50(3-36)$ & $\mathrm{N} / \mathrm{A}$ & 6.80 & $\begin{array}{l}8 \text { weekly aflibercept } \\
\text { injections. No loading } \\
\text { dose }\end{array}$ \\
\hline Cho et $\mathrm{al}^{4 !}$ & $81.00(62-95)$ & $20.20 \pm 7.60(7-37)$ & 28-35 days & $4.40(3-6)$ & $\begin{array}{l}\text { Loading phase of } \\
3 \text { monthly injections, and } \\
\text { then every } 6-8 \text { weeks for } \\
21 \text { eyes }(75 \%)\end{array}$ \\
\hline $\begin{array}{l}\text { de Massougnes } \\
\text { et } \mathrm{al}^{33}\end{array}$ & $78.80 \pm 7.10$ & $26.10 \pm 12.00$ & N/A & $\mathrm{N} / \mathrm{A}$ & $\begin{array}{l}3 \text { monthly loading doses, } \\
\text { and then treat-and- } \\
\text { observe strategy }\end{array}$ \\
\hline Eadie et $\mathrm{a}^{34}$ & 79.90 & $3-38$ & 5.88 weeks $(4-10)$ & $5.53(2-11)$ & $\begin{array}{l}\text { Treat }+ \text { extend approach, } \\
\text { or every } 4 \text { weeks due to } \\
\text { persistent exudation }\end{array}$ \\
\hline Gerding ${ }^{45}$ & $\begin{array}{l}80.60 \pm 7.70 \\
(60.9-100)\end{array}$ & $21.50 \pm 11.70(9-54)$ & 46.5 days & $4.01 \pm 1.14(2-6)$ & PRN \\
\hline
\end{tabular}


Table 2 (Continued)

\begin{tabular}{|c|c|c|c|c|c|}
\hline Study & $\begin{array}{l}\text { Mean age in } \\
\text { years (range) }\end{array}$ & $\begin{array}{l}\text { Number of injections } \\
\text { prior to conversion } \\
\text { (range) }\end{array}$ & $\begin{array}{l}\text { Time between last } \\
\text { anti-VEGF treatment } \\
\text { and conversion (range) }\end{array}$ & $\begin{array}{l}\text { Mean number of } \\
\text { aflibercept injections } \\
\text { (range) }\end{array}$ & Treatment regimen \\
\hline Gharbiya et $\mathrm{al}^{35}$ & $\begin{array}{l}70.10 \pm 8.10 \\
(60-86)\end{array}$ & $34.40 \pm 11.90(15-50)$ & $4.90 \pm 0.80$ weeks $(4-6)$ & $4.50 \pm 1.30(3-6)$ & $\begin{array}{l}\text { Loading dose of } \\
3 \text { injections and } \\
\text { retreatment according to } \\
\text { following factors: I) VA } \\
\text { loss of at least } 5 \text { letters } \\
\text { with OCT evidence of } \\
\text { fluid, 2) persistent or } \\
\text { recurrent IRF/SRF on } \\
\text { OCT, and 3) new SR } \\
\text { hemorrhage from CNV }\end{array}$ \\
\hline Grewal et $\mathrm{al}^{26}$ & $80.70 \pm 4.50$ & $29.80 \pm 17.10(6-70)$ & Not $<28$ days & $10.20 \pm 1.20(10-12)$ & $\begin{array}{l}\text { Loading phase of } \\
3 \text { monthly injections plus } \\
\text { bimonthly injections in } \\
\text { case of resolution of the } \\
\text { edema or monthly in case } \\
\text { of edema }\end{array}$ \\
\hline Hall et $\mathrm{al}^{36}$ & $80.40 \pm 1.45$ & $|4.90 \pm 2.0|(2-53)$ & N/A & $\begin{array}{l}6.27 \pm 0.37 \text { (4-II) } \\
\text { (6-month follow-up: } \\
30 \text { patients received } \\
4.50 \pm 0.11 \text { injections; } \\
9 \text { month follow-up: } \\
26 \text { patients received } \\
6.00 \pm 0.23 ; 12 \text {-month } \\
\text { follow-up: } 22 \text { patients } \\
\text { received } 7.17 \pm 0.38)\end{array}$ & $\begin{array}{l}\text { In the presence of } \\
\text { SRF at the time of the } \\
\text { switch: loading phase of } \\
3 \text { monthly injections, and } \\
\text { then treat-and-extend } \\
\text { regimen. In absence of } \\
\text { SRF at the time of the } \\
\text { switching: treat-and- } \\
\text { extend regimen }\end{array}$ \\
\hline Hariri et $\mathrm{al}^{46}$ & $81.00(60-97)$ & 18.00 & $35.80 \pm 5.04$ days $(28-42)$ & N/A & N/A \\
\hline He et $\mathrm{al}^{47}$ & $82.90 \pm 8.15$ & $17.46 \pm 10.07(3-46)$ & N/A & $7.5 I \pm I .65$ & $\begin{array}{l}82 \text { eyes: standard } \\
\text { therapy: } 3 \text { monthly } \\
\text { loading doses and } \\
\text { bimonthly doses } \\
\text { thereafter } \\
34 \text { eyes: Rx with less } \\
\text { than standard therapy, } \\
\text { undergoing injections } \\
\text { further apart } \\
26 \text { eyes: Rx with more } \\
\text { than standard: monthly } \\
\text { or every } 6 \text { week } \\
\text { injections }\end{array}$ \\
\hline $\begin{array}{l}\text { Kanesa-Thasan } \\
\text { et } \mathrm{al}^{37}\end{array}$ & $80.70 \pm 4.38$ & $25.70 \pm 20.10(6-70)$ & $N / A$ & $\begin{array}{l}15.10 \pm 2.47 \\
18 \text { months }(12-19)\end{array}$ & $\begin{array}{l}\text { Minimum } 3 \text { monthly } \\
\text { aflibercept, and } \\
\text { then switched to a } \\
\text { bimonthly interval if } \\
\text { complete resolution } \\
\text { of SRF or IRF, or kept } \\
\text { at an interval at which } \\
\text { complete resolution } \\
\text { was maintained, based } \\
\text { on treat-and-extend } \\
\text { approach }\end{array}$ \\
\hline Kumar et $\mathrm{al}^{38}$ & $\begin{array}{l}79.00 \pm 8.00 \\
(72-84)\end{array}$ & $28.60 \pm 20.10($ IQR II-43) & $\begin{array}{l}34.40 \pm 5.00 \text { days } \\
\text { (IQR 32-37) }\end{array}$ & $5.30 \pm 0.60$ (IQR 5-6) & $\begin{array}{l}\text { Loading phase of } \\
3 \text { monthly injections } \\
\text { followed by PRN } \\
\text { treatment }\end{array}$ \\
\hline Major et $\mathrm{al}^{48}$ & $81.20(60-95)$ & $24.80(2-66)$ & 36 days $(27-65)$ & N/A & N/A \\
\hline
\end{tabular}


Table 2 (Continued)

\begin{tabular}{|c|c|c|c|c|c|}
\hline Study & $\begin{array}{l}\text { Mean age in } \\
\text { years (range) }\end{array}$ & $\begin{array}{l}\text { Number of injections } \\
\text { prior to conversion } \\
\text { (range) }\end{array}$ & $\begin{array}{l}\text { Time between last } \\
\text { anti-VEGF treatment } \\
\text { and conversion (range) }\end{array}$ & $\begin{array}{l}\text { Mean number of } \\
\text { aflibercept injections } \\
\text { (range) }\end{array}$ & Treatment regimen \\
\hline Mantel et $\mathrm{al}^{27}$ & 76.00 & $\begin{array}{l}20.70 \\
\text { Group A (aflibercept } \\
n=10 \text { eyes): } 21.30 \\
\text { Group R (ranibizumab } \\
n=11 \text { eyes): } 20.10\end{array}$ & $\mathrm{~N} / \mathrm{A}$ & $\begin{array}{l}10.70 \\
\text { Group A } \\
\text { (aflibercept): } 10.70 \\
\text { Group R } \\
\text { (ranibizumab): } 10.70\end{array}$ & $\begin{array}{l}3 \text { monthly injections and } \\
\text { then observe-and-extend } \\
\text { regimen for } 12 \text { months }\end{array}$ \\
\hline $\begin{array}{l}\text { Narayan and } \\
\text { Muecke }^{39}\end{array}$ & $83.20 \pm 7.00$ & $N / A$ & $\mathrm{~N} / \mathrm{A}$ & 7.58 & $\begin{array}{l}48 \text { eyes }(60 \%): 8 \text { weekly; } \\
25 \text { eyes }(31 \%): 6 \text { weekly; } \\
6 \text { eyes }(7.5 \%): 4 \text { weekly; } \\
\text { I eye: initially } 4 \text { weekly } \\
\text { extended to } 12 \text { weekly }\end{array}$ \\
\hline $\begin{array}{l}\text { Pinheiro-Costa } \\
\text { et } \mathrm{al}^{44}\end{array}$ & $76.60(6 I-92)$ & $\begin{array}{l}12.40 \\
16.50(6-24) \text { bevacizumab } \\
\text { group } \\
8.90(3-39) \text { ranibizumab } \\
\text { group }\end{array}$ & $\mathrm{N} / \mathrm{A}$ & $\begin{array}{l}5.10 \pm 1.70 \text { (range } 3-9 \text { ) } \\
\text { Bevacizumab group: } \\
4.50 \pm 1.60 \\
\text { Ranibizumab group: } \\
5.60 \pm 1.60\end{array}$ & Treat and extend \\
\hline Ricci et al ${ }^{49}$ & $\begin{array}{l}79.00 \text { (59-93) } \\
\text { PRN group } \\
77(52-85) \\
\text { fixed regimen } \\
\text { group }\end{array}$ & $\begin{array}{l}5.00(4.5-7.50) \text { PRN group } \\
6.50(4-9.5) \text { fixed regimen } \\
\text { group }\end{array}$ & $\mathrm{N} / \mathrm{A}$ & $\begin{array}{l}3.50 \pm I .60(I-7) P R N \\
\text { group } \\
7.00 \text { fixed regimen group }\end{array}$ & $\begin{array}{l}36 \text { eyes (50\%): PRN } \\
\text { regimen; } 36 \text { eyes ( } 50 \%) \text { : } \\
\text { loading dose of } 3 \text { monthly } \\
\text { injections followed by } \\
\text { fixed bimonthly injection }\end{array}$ \\
\hline Sarao et $\mathrm{a}^{28}$ & $78.30 \pm 8.20$ & $15.20 \pm 1.90$ & $\mathrm{~N} / \mathrm{A}$ & $3.50 \pm 1.80$ & $\begin{array}{l}\text { Initial injection of } \\
\text { aflibercept and then } \\
\text { reviewed every } 4 \text { weeks. } \\
\text { Rx at investigators' } \\
\text { discretion }\end{array}$ \\
\hline Thorell et $\mathrm{al}^{40}$ & $76.20 \pm 8.70$ & $30.70 \pm 15.50$ & $\mathrm{~N} / \mathrm{A}$ & $4.50 \pm 1.00$ & $\begin{array}{l}\text { Treat-and-extend } \\
\text { strategy, starting from } \\
\text { every 4-week treatment }\end{array}$ \\
\hline Wykoff et $\mathrm{al}^{29}$ & $77.80(55-95)$ & $42.00(19-67)$ & 33.30 days (28-68) & 5.60 & $\begin{array}{l}\text { Loading phase of } \\
3 \text { monthly injections, } \\
\text { one mandatory dose at } \\
\text { month } 4 \text { and PRN doses } \\
\text { at months } 3 \text { and } 5\end{array}$ \\
\hline
\end{tabular}

Abbreviations: CNV, choroidal neovascularization; IQR, interquartile range; IRF, intraretinal fluid; N/A, not available; OCT, optical coherence tomography; PRN, pro re nata; $R x$, treatment; SRF, subretinal fluid; SR, subretinal; VA, visual acuity; VEGF, vascular endothelial growth factor.

6 months. Overall, the pooled results showed a significant reduction in CRT from baseline with a mean reduction of $61.90 \mu \mathrm{m}(95 \% \mathrm{CI}-77.10$ to $-46.80, P<0.001)$ (Figure 4).

Eleven studies ( 1,016 eyes) were included in the assessment of CRT change between baseline and 12 months. There was significant reduction in CRT from baseline with a mean reduction of $50.00 \mu \mathrm{m}(95 \% \mathrm{CI}-63.20$ to -36.80 , $P<0.001$ ) (Figure 5). Significant heterogeneity was found between studies assessing CRT for 6 and 12 months of follow-up $\left(I^{2}=97.01 \%, P<0.001\right.$ and $I^{2}=96.95 \%, P<0.001$, respectively).

Subgroup analysis of the number of previous anti-VEGF injections and aflibercept injections throughout the study showed similar mean reduction in CRT across the included studies (Table 4). Those studies incorporating a dosing regimen of 3 loading doses followed by PRN treatment had a mean reduction of -103.20 (at 6 months) and $-95.40 \mu \mathrm{m}$ (at 12 months) compared to those studies incorporating a dosing regimen of 3 loading doses followed by treat-and-extend treatment which had a mean reduction of -38.40 ( 6 months) and $-24.00 \mu \mathrm{m}$ (12 months). There was an initial similar difference between study designs, with prospective studies illustrating a greater CRT reduction of -70.00 (6 months) and $-85.50 \mu \mathrm{m}$ (12 months) compared to those retrospective in nature illustrating -58.30 (6 months) and $-38.80 \mu \mathrm{m}$ (12 months).

\section{PED height}

Eight studies (474 eyes) were included in the analysis of mean change in PED height for 6 months of follow-up. There was a significant reduction in PED height from 


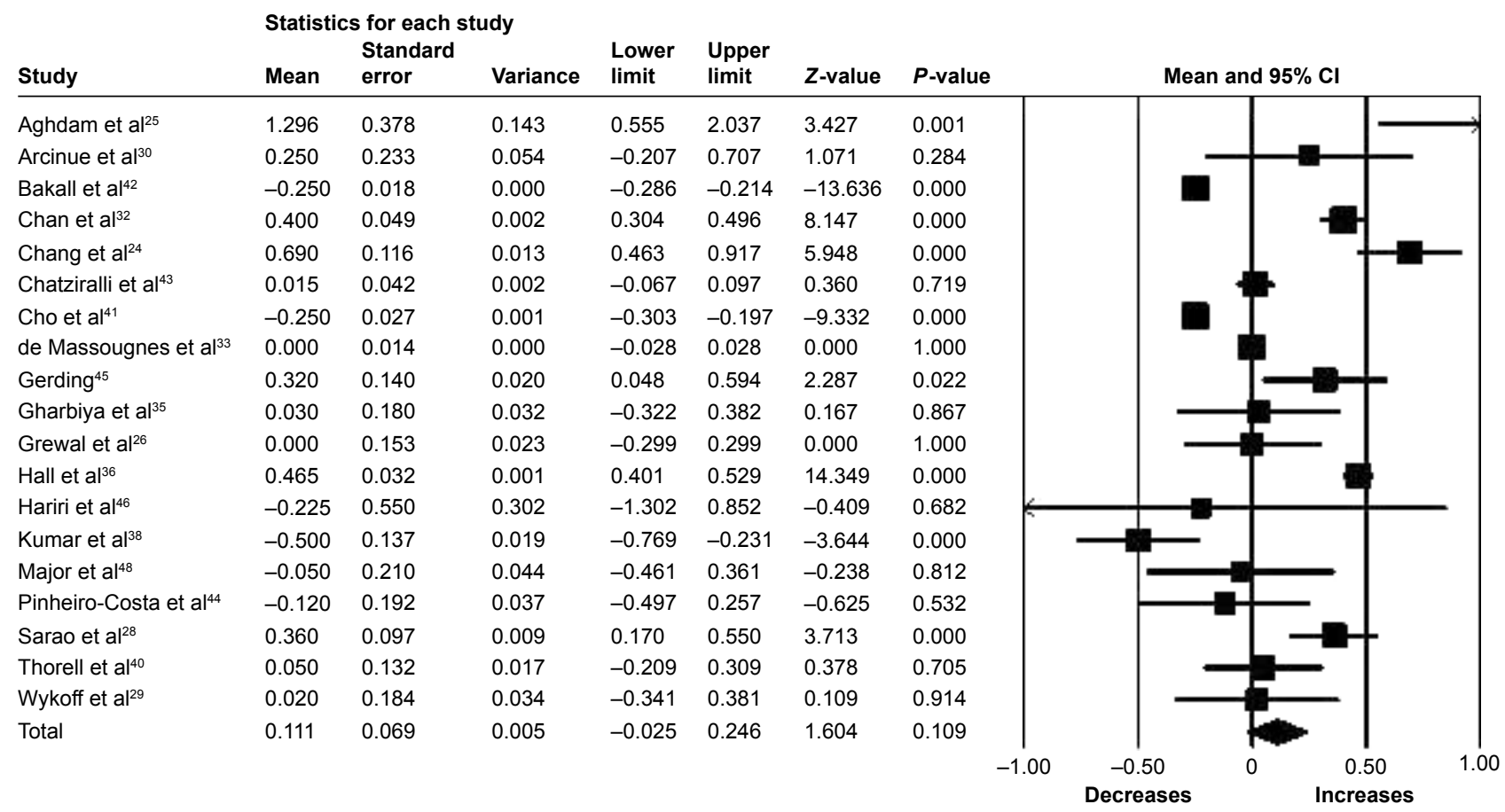

Figure 2 Forest plot of 19 studies reporting results of best-corrected visual acuity 6 months after the switch to aflibercept.

Note: Best-corrected visual acuity was divided by 10 for better visualization of the forest plot.

Abbreviation: $\mathrm{Cl}$, confidence interval.

baseline with a mean reduction of $51.20 \mu \mathrm{m}(95 \% \mathrm{CI}-55.10$ to $-47.40, P<0.001)$ (Figure 6$)$. Heterogeneity $\left(I^{2}=0 \%\right.$, $P=0.69)$ was not detected between studies in this analysis.

There was no difference in PED height change across the different dosing regimens and among the study designs (data not shown).

\section{Ocular and systemic adverse events}

Reported ocular and systemic adverse events were rare and only reported in 8 of the studies. Another 13 studies indicated that systemic and ocular adverse events were being monitored but with no reported incidence of adverse events. Only a small number of ocular adverse events were reported; among

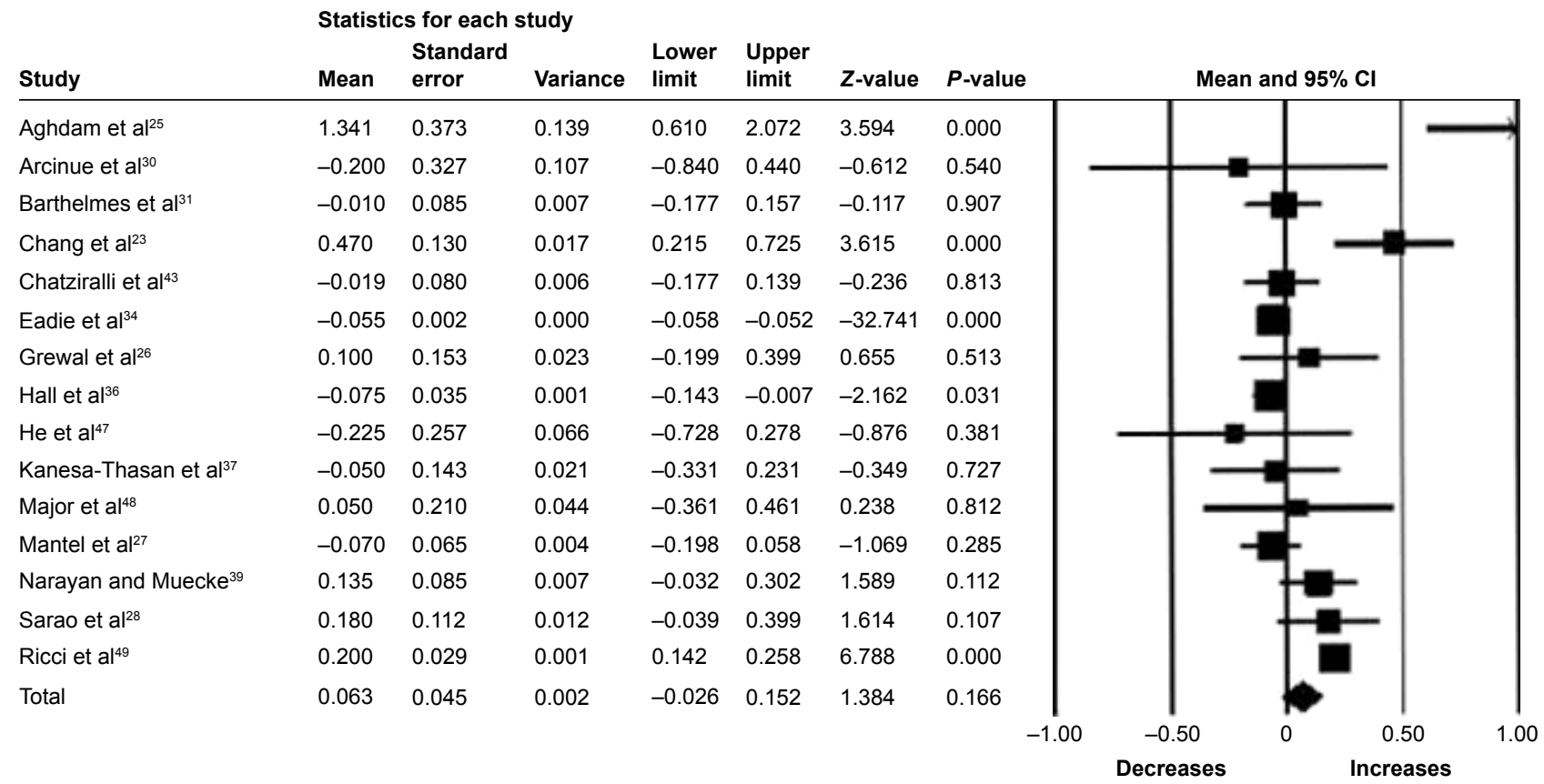

Figure 3 Forest plot of 15 studies reporting results of best-corrected visual acuity 12 months after the switch to aflibercept.

Note: Best-corrected visual acuity was divided by 10 for better visualization of the forest plot.

Abbreviation: $\mathrm{Cl}$, confidence interval. 


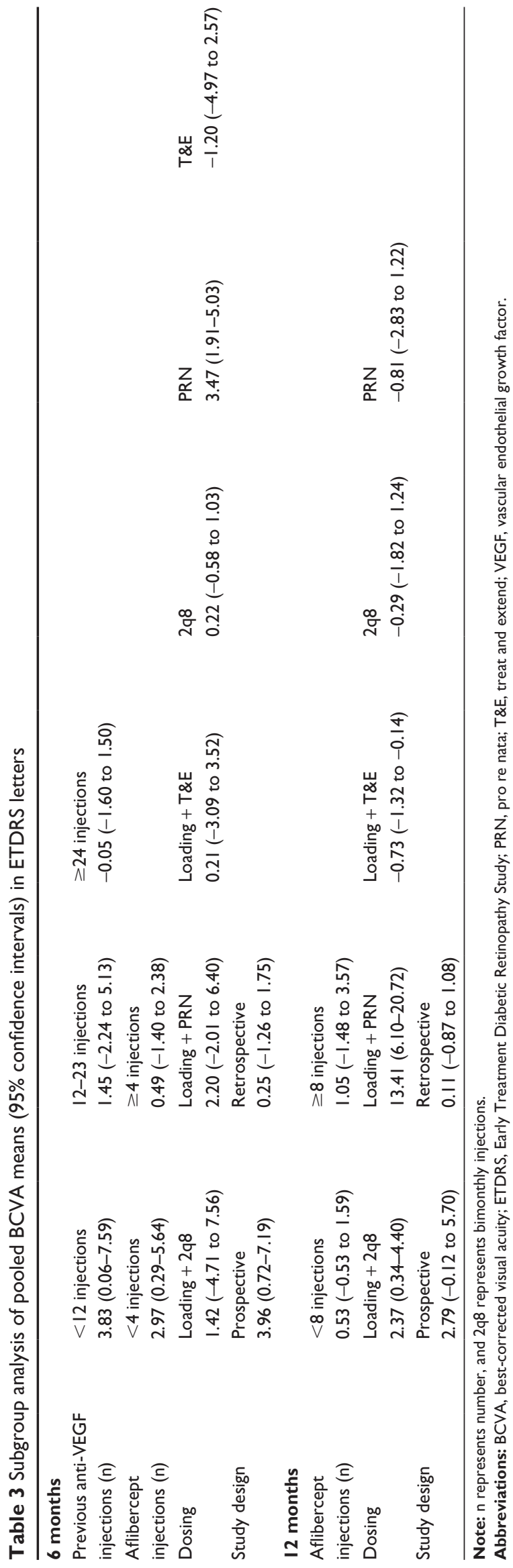

those, endophthalmitis rates were low with 3 reported cases in the cohort of 2,254 eyes $(<1 \%)$. There were $4(<1 \%)$ reported cases of cataract or lens changes, 3 eyes $(<1 \%)$ with progression of GA, $3(<1 \%)$ eyes which developed a tear, 2 eyes $(<1 \%)$ with subretinal hemorrhage, 2 eyes $(<1 \%)$ with submacular hemorrhage, 1 eye $(<1 \%)$ with retinal detachment, and 3 eyes with intraocular pressure increase $(<1 \%)$.

\section{Publication bias}

There was evidence of possible publication bias when assessed by the funnel plots and by Egger's test $(P=0.30 \mathrm{BCVA}$ 6 months; $P=0.005$ BCVA 12 months; $P=0.06$ CRT 6 months; $P=0.02$ CRT 12 months; $P=0.43$ PED 6 months), indicating that the treatment effect was overestimated as majority of studies were having small population sample.

\section{Discussion}

In this review, a meta-analysis was used to analyze the efficacy of switching to aflibercept monotherapy in clinical practice among patients with treatment-resistant AMD. The results demonstrated considerable improvement in anatomical outcomes, though functional improvement was limited. Overall, our results show that treatment-resistant patients may benefit from switching to aflibercept in reducing retinal thickness and maintaining visual function.

Although there were significant morphological improvements in terms of CRT and PED height, there were no comparative responses in terms of visual function. The absence of an associated improvement in visual acuity could be due to multiple factors, including better baseline visual acuity, greater foveal thickness, and angiographic lesion type and size ${ }^{50-52}$ limiting the potential for improvement. The chronic nature of nAMD in these patients may progress to atrophic changes ${ }^{12,53,54}$ and subsequent photoreceptor and RPE damage ${ }^{55}$ leading to a possible pharmacological ceiling effect. The CATT trials showed an increase in GA with monthly ranibizumab injections; ${ }^{55}$ thus, it has been suggested aflibercept's higher binding affinity for VEGF may aggravate preexisting GA in switching patients. ${ }^{56}$

Furthermore, the chronicity of disease is demonstrated among eyes which received more injections prior to the switch to aflibercept having poorer outcomes than those eyes with fewer previous injections. Additionally, eyes receiving more injections of aflibercept during the study period had minimal improvement in BCVA and CRT reflecting the treatment-resistant nature of this population. Subsequently, a decreased effect over time is suggestive of 


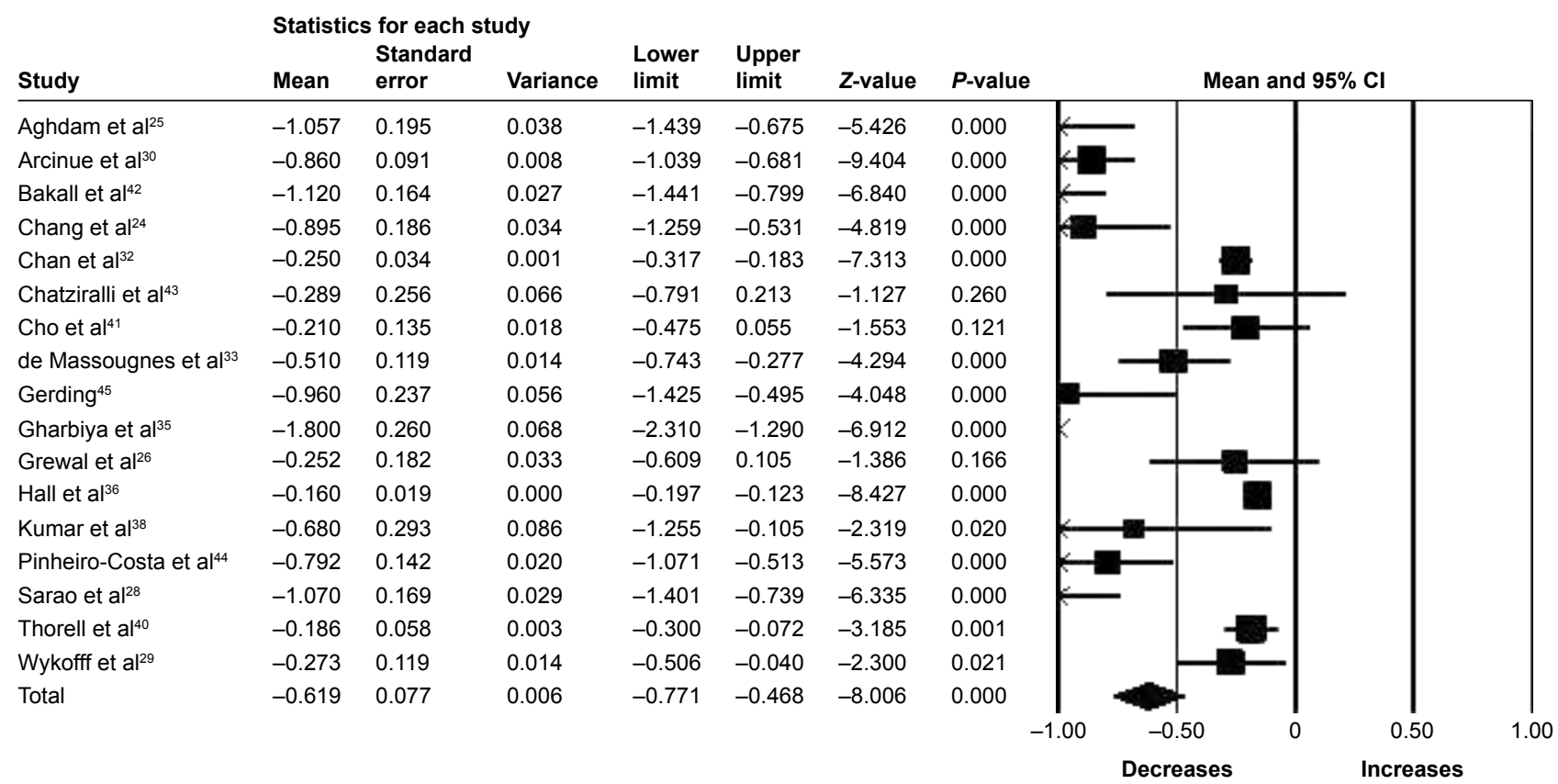

Figure 4 Forest plot of 17 studies reporting results of central retinal thickness 6 months after the switch to aflibercept.

Note: Central retinal thickness was divided by 100 for better visualization of the forest plot.

Abbreviation: $\mathrm{Cl}$, confidence interval.

a drug tolerance as VEGF continues to be expressed as more therapy is administered. ${ }^{57}$

Prospective studies assessing the switch to aflibercept demonstrated better functional and anatomical improvements than the retrospective studies. Prospective studies typically have a more structured protocol with predefined time points, thus reducing known biases incurred from retrospective study designs. ${ }^{58}$ The window for visit time-points among retrospective studies may be highly variable, leading to measurement bias.

The pooled results highlight the benefit of a loading dose; the studies which did not include a loading dose did not improve comparatively to those that did. ${ }^{59}$ This may be a reflection of the chronicity of the disease among these patients requiring more intensive treatment. Few of the included studies reported the frequency of scheduled visits

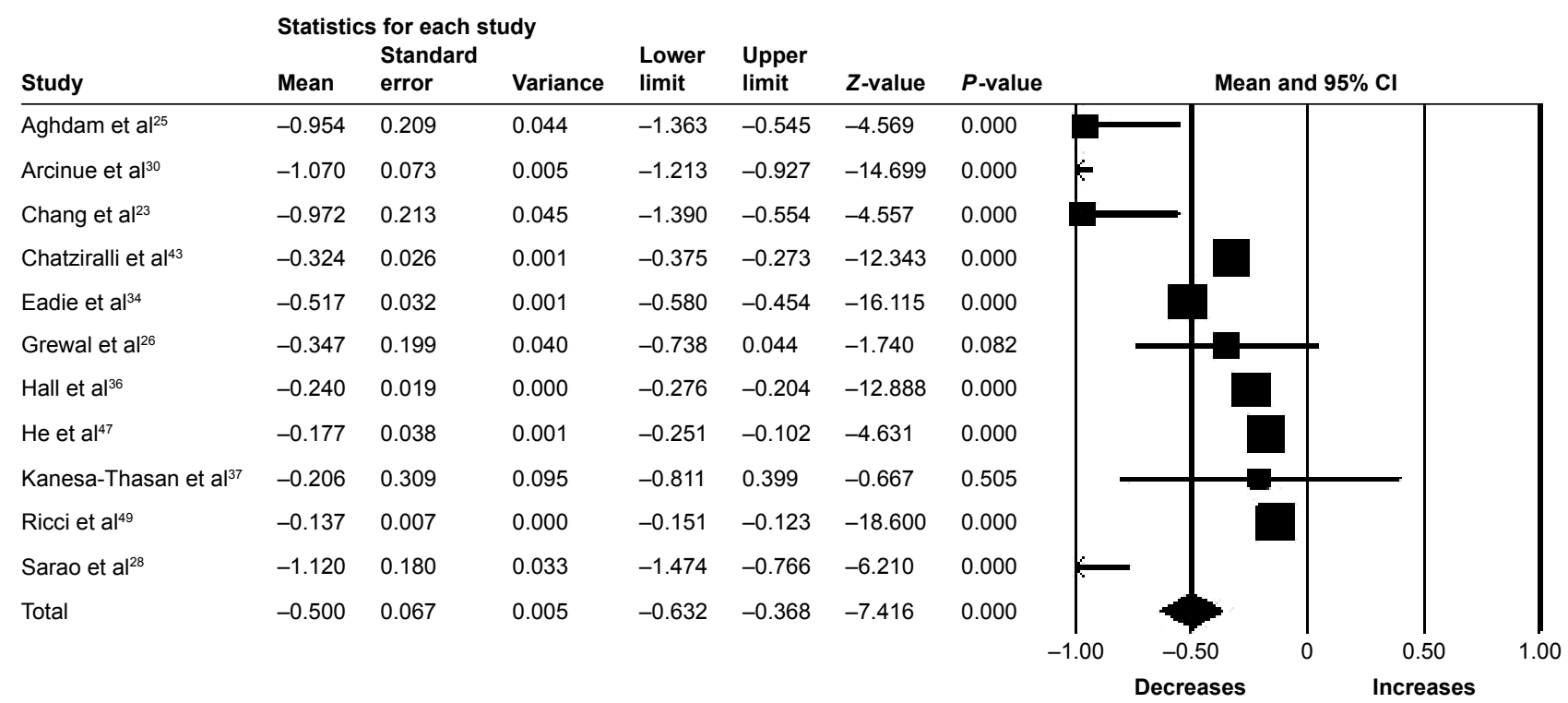

Figure 5 Forest plot of II studies reporting results of central retinal thickness 12 months after the switch to aflibercept.

Note: Central retinal thickness was divided by 100 for better visualization of the forest plot.

Abbreviation: $\mathrm{Cl}$, confidence interval. 


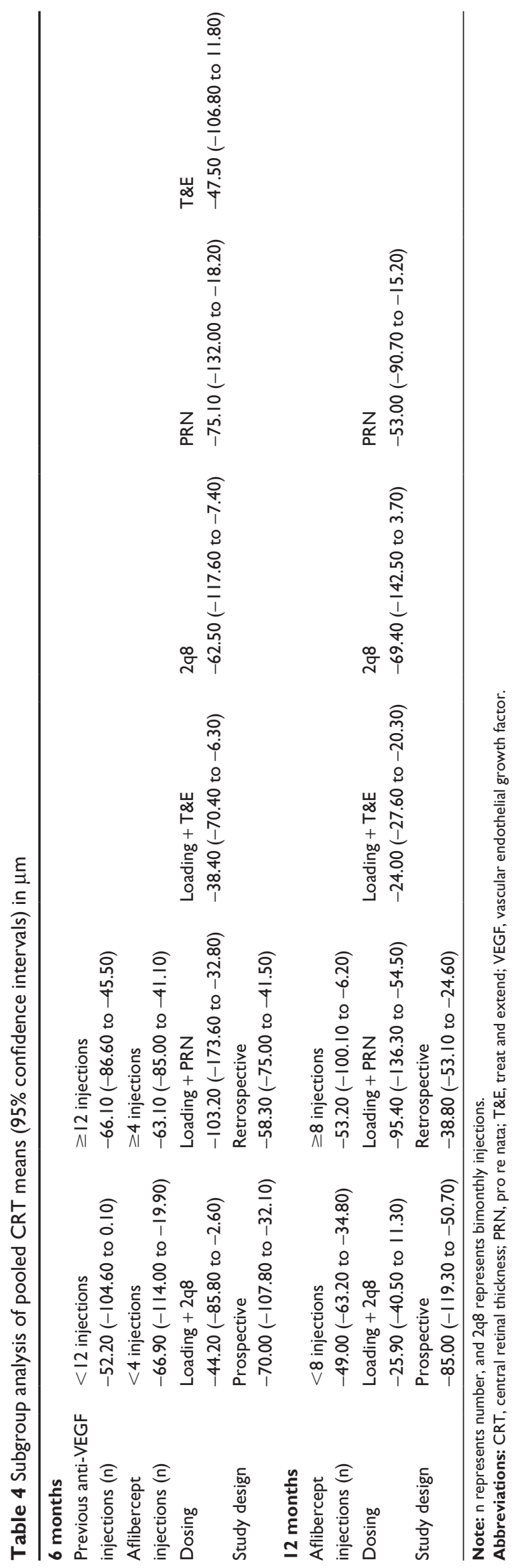

throughout the study period; however, those which did reported monthly follow-up visits supporting the need for more intensive monitoring.

Studies that administered PRN dosing improved more compared to those which administered bimonthly injections over the same period. This is contrary to the VIEW studies among naïve eyes, which did not find any long-term detrimental effects despite the sawtooth pattern of the optical coherence tomography thickness in the bimonthly aflibercept arm. ${ }^{60,61}$ The number of aflibercept injections administered among the PRN group (range 4.50-5.72) regimens compared to the bimonthly group (range 4.40-5.00) regimens was comparable over the 6 months. Among the studies which had a PRN regimen, only 1 described the number of injections (7.27) over 12 months, and studies which had bimonthly injection had between 5.25 and 10.20 injections. Similarly, PRN dosing regimen displayed the most improvement in contrast to those studies following a loading followed by treat-andextend regimen (range of injections 3.14-5.30), reflecting the need for closer monitoring in these resistant patients. The slightly higher number of injections within the PRN group may indicate the resistant nature of these eyes, with the majority needing more frequent injections than the bimonthly dosing recommended by the manufacturer. However, it has been reported that aflibercept does not maintain its effect for 2 months in many patients, as $79 \%$ of eyes in the subjects with exudative AMD who were incomplete responders to multiple ranibizumab anti-VEGF injections (TURF trials) required treatment on PRN visits, ${ }^{29}$ indicative of the resistant nature of these patients. The pooled results suggest an individualized PRN treatment regime may yield better results.

The safety profile of aflibercept was similar to bevacizumab and ranibizumab based on information in the included studies. ${ }^{15,62}$ We reported the outcomes of 28 papers for a total of 2,254 eyes. Although the reporting of adverse events was inconsistent, most of the included studies reported as "no systemic or ocular adverse events occurred". Among studies which reported adverse events, endophthalmitis and retinal detachment rates were low $(<1 \%)$.

Several other therapeutic approaches have been suggested to manage treatment resistance in these patients: increasing the frequency between treatment intervals; ${ }^{63,64}$ increasing the dose of the same anti-VEGF therapy; ${ }^{63,65}$ and combining anti-VEGF therapy with another treatment modality, such as verteporfin PDT. ${ }^{66}$ However, switching to a different anti-VEGF agent, such as aflibercept, seems to have the best effect in terms of decreasing CRT, PED height, and the reduction in retinal fluid. 


\begin{tabular}{|c|c|c|c|c|c|c|c|c|c|}
\hline Study & $\begin{array}{l}\text { Statisti } \\
\text { Mean }\end{array}$ & $\begin{array}{l}s \text { for each s } \\
\text { Standard } \\
\text { error }\end{array}$ & $\begin{array}{l}\text { udy } \\
\text { Variance }\end{array}$ & $\begin{array}{l}\text { Lower } \\
\text { limit }\end{array}$ & $\begin{array}{l}\text { Upper } \\
\text { limit }\end{array}$ & $Z$-value & $P$-value & \multicolumn{2}{|c|}{ Mean and $95 \% \mathrm{Cl}$} \\
\hline Bakall et $a^{42}$ & -0.520 & 0.022 & 0.000 & -0.562 & -0.478 & -24.000 & 0.000 & & \\
\hline Broadhead et a ${ }^{22}$ & -0.515 & 0.125 & 0.016 & -0.760 & -0.271 & -4.134 & 0.000 & & \\
\hline Chan et al ${ }^{32}$ & -0.434 & 0.078 & 0.006 & -0.587 & -0.281 & -5.545 & 0.000 & - & \\
\hline de Massougnes et al ${ }^{13}$ & -0.380 & 0.159 & 0.025 & -0.691 & -0.069 & -2.393 & 0.017 & & \\
\hline Gharbiya et al $\left.\right|^{35}$ & -0.790 & 0.180 & 0.032 & -1.142 & -0.438 & -4.399 & 0.000 & 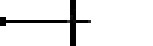 & \\
\hline Grewal et $a^{26}$ & -0.405 & 0.319 & 0.102 & -1.030 & 0.221 & -1.268 & 0.205 & & \\
\hline Kumar et $a^{38}$ & -0.450 & 0.244 & 0.059 & -0.927 & 0.027 & -1.848 & 0.065 & & \\
\hline Major et al ${ }^{48}$ & -0.470 & 0.086 & 0.007 & -0.638 & -0.302 & -5.475 & 0.000 & & \\
\hline Total & -0.512 & 0.020 & 0.000 & -0.551 & -0.474 & -26.063 & 0.000 & & \\
\hline & & & & & & & -1.00 & $\begin{array}{c}-0.50 \\
\text { Decreases }\end{array}$ & $\begin{array}{c}0.50 \\
\text { Increases }\end{array}$ \\
\hline
\end{tabular}

Figure 6 Forest plot of 8 studies reporting results of pigment epithelial detachment height 6 months after the switch to aflibercept. Note: Pigment epithelium detachment values were divided by 100 for better visualization of the forest plot.

Abbreviation: $\mathrm{Cl}$, confidence interval.

This meta-analysis validates the effectiveness of aflibercept in improving morphology in patients with AMD refractory to ranibizumab and bevacizumab. ${ }^{67,68}$ This may be because aflibercept binds VEGF-A, VEGF-B, and PIGF, while other anti-VEGFs only bind with VEGF-A. Aflibercept's higher binding affinity than bevacizumab and ranibizumab may attribute to this better outcome. ${ }^{67}$ Using aflibercept for those with treatment resistance could potentially inhibit more angiogenic factors improving efficacy.

The results of this meta-analysis should be interpreted with caution for several reasons. Firstly, the available studies generally contained small numbers of participants, which may lead to an overestimation of treatment effect among the individual studies which may accentuate the results of this meta-analysis. Secondly, a majority of the studies were retrospective in nature, and there were no randomized trials to accommodate for confounding variables. Thirdly, there was considerable variability among study designs and dosing regimens.

\section{Conclusion}

This analysis shows that patients with nAMD, who have had an inadequate response to ranibizumab and/or bevacizumab, may benefit by switching to aflibercept therapy.

Of importance, the eyes included in this study were poor responders and had limited potential for visual recovery as the disease is both chronic and advanced in nature; nonetheless, they still displayed an improvement. Although vision did not significantly improve, vision stability may have an immense impact on the patient's quality of life in terms of activities of daily living, independence, and mobility and decrease the risk of comorbidities such as depression. ${ }^{69}$ As these difficult-to-treat patients are likely to have more chronic disease, recommended bimonthly treatment for naïve eyes may not be adequate for this group. Changing to aflibercept with a loading dose and PRN regimen on an individualized basis may prove to be an effective management strategy.

\section{Acknowledgment}

Bayer Corporation Global provided financial support via unrestricted research grant. The sponsor had no role in the design or conduct of this research.

\section{Disclosure}

Andrew A Chang is a consultant for Alcon, Bayer, and Novartis. Kimberly Spooner, Thomas Hong, and Wijeyanthy Wijeyakumar report no conflicts of interest in this work.

\section{References}

1. Friedman DS, O'Colmain BJ, Muñoz B, et al; Eye Diseases Prevalence Research Group. Prevalence of age-related macular degeneration in the United States. Arch Ophthalmol. 2004;122(4):564-572.

2. Klaver CC, Wolfs RC, Vingerling JR, Hofman A, de Jong PT. Agespecific prevalence and causes of blindness and visual impairment in an older population: the Rotterdam study. Arch Ophthalmol. 1998;116(5): 653-658.

3. Wang JJ, Foran S, Mitchell P. Age-specific prevalence and causes of bilateral and unilateral visual impairment in older Australians: the Blue Mountains Eye Study. Clin Exp Ophthalmol. 2000;28(4):268-273.

4. Wong WL, Su X, Li X, et al. Global prevalence of age-related macular degeneration and disease burden projection for 2020 and 2040: a systematic review and meta-analysis. Lancet Glob Health. 2014;2(2): e106-e116.

5. Resnikoff S, Pascolini D, Etya'ale D, et al. Global data on visual impairment in the year 2002. Bull World Health Organ. 2004;82(11):844-851.

6. Gehrs KM, Anderson DH, Johnson LV, Hageman GS. Age-related macular degeneration - emerging pathogenetic and therapeutic concepts. Ann Med. 2006;38(7):450-471. 
7. Bressler NM. Age-related macular degeneration is the leading cause of blindness. JAMA. 2004;291(15):1900-1901.

8. Emerson MV, Lauer AK. Current and emerging therapies for the treatment of age-related macular degeneration. Clin Ophthalmol. 2008;2(2):377-388.

9. U.S. National Institutes of Health. Current studies: dry macular degeneration. Available from: https://clinicaltrials.gov/ct2/results?term= dry+macular+degeneration\&Search=Search. Accessed July 7, 2016.

10. Wang H, Hartnett ME. Regulation of signaling events involved in the pathophysiology of neovascular AMD. Mol Vis. 2016;22:189-202.

11. Wang L, Lee AY, Wigg JP, Peshavariya H, Liu P, Zhang H. miR-126 regulation of angiogenesis in age-related macular degeneration in $\mathrm{CNV}$ mouse model. Int J Mol Sci. 2016;17(6).

12. Jager RD, Mieler WF, Miller JW. Age-related macular degeneration. $N$ Engl J Med. 2008;358(24):2606-2617.

13. U.S. Food \& Drug Administration. FDA approves new biologic treatment for wet age-related macular degeneration. 2006. Available from: http:// www.fda.gov/NewsEvents/Newsroom/PressAnnouncements/2006/ ucm108685.htm. Accessed July 18, 2016.

14. US Food \& Drug Administration. FDA approves Eylea for eye disorder in older people. 2011. Available from: http://www.fda.gov/NewsEvents/ Newsroom/PressAnnouncements/ucm280601.htm. Accessed July 18, 2016.

15. Heier JS, Brown DM, Chong V, et al; VIEW 1 and VIEW 2 Study Groups. Intravitreal aflibercept (VEGF trap-eye) in wet age-related macular degeneration. Ophthalmology. 2012;119(12):2537-2548.

16. Ehlken C, Jungmann S, Böhringer D, Agostini HT, Junker B, Pielen A. Switch of anti-VEGF agents is an option for nonresponders in the treatment of AMD. Eye (Lond). 2014;28(5):538-545

17. Binder S. Loss of reactivity in intravitreal anti-VEGF therapy: tachyphylaxis or tolerance? Br J Ophthalmol. 2012;96(1):1-2.

18. Broadhead GK, Hong T, Chang AA. Treating the untreatable patient: current options for the management of treatment-resistant neovascular age-related macular degeneration. Acta Ophthalmol. 2014;92(8): 713-723.

19. Higgins JP, Thompson SG, Deeks JJ, Altman DG. Measuring inconsistency in meta-analyses. BMJ. 2003;327(7414):557-560.

20. Downs SH, Black N. The feasibility of creating a checklist for the assessment of the methodological quality both of randomised and nonrandomised studies of health care interventions. J Epidemiol Community Health. 1998;52(6):377-384.

21. Higgins JP, Thompson SG. Quantifying heterogeneity in a metaanalysis. Stat Med. 2002;21(11):1539-1558.

22. Broadhead GK, Hong T, Zhu M, et al. Response of pigment epithelial detachments to intravitreal aflibercept among patients with treatmentresistant neovascular age-related macular degeneration. Retina. 2015; 35(5):975-981.

23. Chang AA, Broadhead GK, Hong T, et al. Intravitreal aflibercept for treatment-resistant neovascular age-related macular degeneration: 12-month safety and efficacy outcomes. Ophthalmic Res. 2015;55(2): 84-90.

24. Chang AA, Li H, Broadhead GK, et al. Intravitreal aflibercept for treatment-resistant neovascular age-related macular degeneration. Ophthalmology. 2014;121(1):188-192.

25. Aghdam KA, Pielen A, Framme C, Junker B. Visual and anatomic outcomes after conversion to aflibercept in neovascular age-related macular degeneration: 12-month results. Eur J Ophthalmol. 2016; 26(5):473-478.

26. Grewal DS, Gill MK, Sarezky D, Lyon AT, Mirza RG. Visual and anatomical outcomes following intravitreal aflibercept in eyes with recalcitrant neovascular age-related macular degeneration: 12-month results. Eye (Lond). 2014;28(7):895-899.

27. Mantel I, Gianniou C, Dirani A. Conversion to aflibercept therapy versus continuing with ranibizumab therapy for neovascular age-related macular degeneration dependent on monthly ranibizumab treatment. Retina. 2016;36(1):53-58.
28. Sarao V, Parravano M, Veritti D, Arias L, Varano M, Lanzetta P. Intravitreal aflibercept for chorioidal neovascularization due to age-related macular degeneration unresponsive to ranibizumab therapy. Retina. 2016;36(4):770-777.

29. Wykoff CC, Brown DM, Maldonado ME, Croft DE. Aflibercept treatment for patients with exudative age-related macular degeneration who were incomplete responders to multiple ranibizumab injections (TURF trial). Br J Ophthalmol. 2014;98(7):951-955.

30. Arcinue CA, Ma F, Barteselli G, Sharpsten L, Gomez ML, Freeman WR. One-year outcomes of aflibercept in recurrent or persistent neovascular age-related macular degeneration. Am J Ophthalmol. 2015;159(3): 426-436.e2.

31. Barthelmes D, Campain A, Nguyen P, et al; Fight Retinal Blindness! Project Investigators. Effects of switching from ranibizumab to aflibercept in eyes with exudative age-related macular degeneration. $\mathrm{Br} J$ Ophthalmol. 2016;100(12):1640-1645.

32. Chan CK, Jain A, Sadda S, Varshney N. Optical coherence tomographic and visual results at 6 months after transitioning to aflibercept for patients on prior ranibizumab or bevacizumab treatment for exudative age-related macular degeneration (an American Ophthalmological Society thesis). Trans Am Ophthalmol Soc. 2014;112:160-198.

33. de Massougnes S, Dirani A, Ambresin A, Decugis D, Marchionno L, Mantel I. Pigment epithelial detachment response to aflibercept in neovascular age-related macular degeneration refractory to ranibizumab: time course and drug effects. Retina. 2016;36(5):881-888.

34. Eadie JA, Gottlieb JL, Ip MS, et al. Response to aflibercept in patients with persistent exudation despite prior treatment with bevacizumab or ranibizumab for age-related macular degeneration. Ophthalmic Surg Lasers Imaging Retina. 2014;45(5):394-397.

35. Gharbiya M, Iannetti L, Parisi F, De Vico U, Mungo ML, Marenco M. Visual and anatomical outcomes of intravitreal aflibercept for treatmentresistant neovascular age-related macular degeneration. BioMed Res Int. 2014;2014:273754.

36. Hall LB, Zebardast N, Huang JJ, Adelman RA. Aflibercept in the treatment of neovascular age-related macular degeneration in previously treated patients. J Ocul Pharmacol Ther. 2014;30(4):346-352.

37. Kanesa-Thasan A, Grewal DS, Gill MK, Lyon AT, Mirza RG. Quantification of change in pigment epithelial detachment volume and morphology after transition to intravitreal aflibercept in eyes with recalcitrant neovascular AMD: 18-month results. Ophthalmic Surg Lasers Imaging Retina. 2015;46(6):638-641.

38. Kumar N, Marsiglia M, Mrejen S, et al. Visual and anatomical outcomes of intravitreal aflibercept in eyes with persistent subfoveal fluid despite previous treatments with ranibizumab in patients with neovascular agerelated macular degeneration. Retina. 2013;33(8):1605-1612.

39. Narayan DS, Muecke J. Intravitreal aflibercept treatment in eyes with exudative age-related macular degeneration following prior treatment with intravitreal ranibizumab. Indian J Ophthalmol. 2015;63(11): 832-836.

40. Thorell MR, Nunes RP, Chen GW, et al. Response to aflibercept after frequent re-treatment with bevacizumab or ranibizumab in eyes with neovascular AMD. Ophthalmic Surg Lasers Imaging Retina. 2014; 45(6):526-533.

41. Cho H, Shah CP, Weber M, Heier JS. Aflibercept for exudative AMD with persistent fluid on ranibizumab and/or bevacizumab. $\mathrm{Br} J$ Ophthalmol. 2013;97(8):1032-1035.

42. Bakall B, Folk JC, Boldt HC, et al. Aflibercept therapy for exudative age-related macular degeneration resistant to bevacizumab and ranibizumab. Am J Ophthalmol. 2013;156(1):15-22.e1.

43. Chatziralli I, Nicholson L, Vrizidou E, et al. Predictors of outcome in patients with neovascular age-related macular degeneration switched from ranibizumab to 8-weekly aflibercept. Ophthalmology. 2016;123(8): $1762-1770$

44. Pinheiro-Costa J, Costa JM, Beato JN, et al. Switch to aflibercept in the treatment of neovascular AMD: one-year results in clinical practice. Ophthalmologica. 2015;233(3-4):155-161. 
45. Gerding H. Functional and anatomic efficacy of a conversion to aflibercept in eyes with age-related macular degeneration after long-term ranibizumab treatment. Klin Monbl Augenheilkd. 2015;232(4):560-563.

46. Hariri A, Diniz B, Fou LV, Lam LA, Nittala MG, Sadda SR. Quantitative OCT subanalysis of eyes with choroidal neovascularization switched from multiple injections of bevacizumab or ranibizumab to intravitreal aflibercept. Ophthalmic Surg Lasers Imaging Retina. 2015;46(2): 195-200.

47. He L, Silva RA, Ayoub N, Moshfeghi DM, Leng T. Experience with aflibercept for the treatment of neovascular age-related macular degeneration. Ophthalmic Surg Lasers Imaging Retina. 2015;46(5):542-549.

48. Major JC Jr, Wykoff CC, Croft DE, et al. Aflibercept for pigment epithelial detachment for previously treated neovascular age-related macular degeneration. Can J Ophthalmol. 2015;50(5):373-377.

49. Ricci F, Parravano M, Regine F, et al. Aflibercept in persistent neovascular AMD: comparison of different treatment strategies in switching therapy. Eye (Lond). 2016;30(8):1077-1083.

50. Francis PJ. The influence of genetics on response to treatment with ranibizumab (Lucentis) for age-related macular degeneration: the Lucentis Genotype Study (an American Ophthalmological Society thesis). Trans Am Ophthalmol Soc. 2011;109:115-156.

51. de Oliveira Dias JR, Rodrigues EB, Maia M, Magalhães O Jr, Penha FM, Farah ME. Cytokines in neovascular age-related macular degeneration: fundamentals of targeted combination therapy. Br J Ophthalmol. 2011;95(12):1631-1637.

52. Krebs I, Glittenberg C, Ansari-Shahrezaei S, Hagen S, Steiner I, Binder S. Non-responders to treatment with antagonists of vascular endothelial growth factor in age-related macular degeneration. Br J Ophthalmol. 2013;97(11):1443-1446.

53. Moutray T, Alarbi M, Mahon G, Stevenson M, Chakravarthy U. Relationships between clinical measures of visual function, fluorescein angiographic and optical coherence tomography features in patients with subfoveal choroidal neovascularisation. Br J Ophthalmol. 2008; 92(3):361-364.

54. Martin DF, Maguire MG, Fine SL, et al; Comparison of Age-related Macular Degeneration Treatments Trials (CATT) Research Group. Ranibizumab and bevacizumab for treatment of neovascular agerelated macular degeneration: two-year results. Ophthalmology. 2012; 119(7):1388-1398.

55. Grunwald JE, Daniel E, Huang J, et al; CATT Research Group. Risk of geographic atrophy in the comparison of age-related macular degeneration treatments trials. Ophthalmology. 2014;121(1):150-161.

56. Cheng S, Leng T. Factors associated with poor response to aflibercept after switching from ranibizumab or bevacizumab in neovascular agerelated macular degeneration. Ophthalmic Surg Lasers Imaging Retina. 2016;47(5):458-465
57. Yang S, Zhao J, Sun X. Resistance to anti-VEGF therapy in neovascular age-related macular degeneration: a comprehensive review. Drug Des Devel Ther. 2016;10:1857-1867.

58. Euser AM, Zoccali C, Jager KJ, Dekker FW. Cohort studies: prospective versus retrospective. Nephron Clin Pract. 2009;113(3):c214-c217.

59. Menon G, Chandran M, Sivaprasad S, Chavan R, Narendran N, Yang Y. Is it necessary to use three mandatory loading doses when commencing therapy for neovascular age-related macular degeneration using bevacizumab? (BeMOc Trial). Eye (Lond). 2013;27(8):959-963.

60. Richard G, Monés J, Wolf S, et al. Scheduled versus Pro Re Nata Dosing in the VIEW trials. Ophthalmology. 2015;122(12):2497-2503.

61. Freund KB, Engelbert M, Fine HF. Individualizing the intravitreal antiVEGF dosing regimen for long-term management of neovascular AMD. Ophthalmic Surg Lasers Imaging Retina. 2015;46(5):508-512.

62. Schmidt-Erfurth U, Kaiser PK, Korobelnik JF, et al. Intravitreal aflibercept injection for neovascular age-related macular degeneration: ninetysix-week results of the VIEW studies. Ophthalmology. 2014;121(1): 193-201.

63. Gasperini JL, Fawzi AA, Khondkaryan A, et al. Bevacizumab and ranibizumab tachyphylaxis in the treatment of choroidal neovascularisation. Br J Ophthalmol. 2012;96(1):14-20.

64. Forooghian F, Cukras C, Meyerle CB, Chew EY, Wong WT. Tachyphylaxis after intravitreal bevacizumab for exudative age-related macular degeneration. Retina. 2009;29(6):723-731.

65. Schaal S, Kaplan HJ, Tezel TH. Is there tachyphylaxis to intravitreal anti-vascular endothelial growth factor pharmacotherapy in age-related macular degeneration? Ophthalmology. 2008;115(12):2199-2205.

66. Wang W, He M, Zhang X. Combined intravitreal anti-VEGF and photodynamic therapy versus photodynamic monotherapy for polypoidal choroidal vasculopathy: a systematic review and meta-analysis of comparative studies. PLoS One. 2014;9(10):e110667.

67. Lazzeri S, Ripandelli G, Sartini MS, et al. Aflibercept administration in neovascular age-related macular degeneration refractory to previous anti-vascular endothelial growth factor drugs: a critical review and new possible approaches to move forward. Angiogenesis. 2015;18(4):397-432.

68. Seguin-Greenstein S, Lightman S, Tomkins-Netzer O. A meta-analysis of studies evaluating visual and anatomical outcomes in patients with treatment resistant neovascular age-related macular degeneration following switching to treatment with aflibercept. J Ophthalmol. 2016; 2016:4095852

69. Zhu M, Wijeyakumar W, Syed AR, et al. Vision-related quality of life: 12-month aflibercept treatment in patients with treatment-resistant neovascular age-related macular degeneration. Graefes Arch Clin Exp Ophthalmol. Epub 2016 Aug 30.
Clinical Ophthalmology

\section{Publish your work in this journal}

Clinical Ophthalmology is an international, peer-reviewed journal covering all subspecialties within ophthalmology. Key topics include: Optometry; Visual science; Pharmacology and drug therapy in eye diseases; Basic Sciences; Primary and Secondary eye care; Patient Safety and Quality of Care Improvements. This journal is indexed on Submit your manuscript here: http://www.dovepress.com/clinical-ophthalmology-journal

\section{Dovepress}

PubMed Central and CAS, and is the official journal of The Society of Clinical Ophthalmology (SCO). The manuscript management system is completely online and includes a very quick and fair peer-review system, which is all easy to use. Visit http://www.dovepress.com/ testimonials.php to read real quotes from published authors. 